\title{
The Theory of Multidimensional Persistence
}

\author{
Gunnar Carlsson · Afra Zomorodian
}

Received: 1 October 2007 / Revised: 2 March 2009 / Accepted: 2 March 2009 /

Published online: 24 April 2009

(C) Springer Science+Business Media, LLC 2009

\begin{abstract}
Persistent homology captures the topology of a filtration-a one-parameter family of increasing spaces-in terms of a complete discrete invariant. This invariant is a multiset of intervals that denote the lifetimes of the topological entities within the filtration. In many applications of topology, we need to study a multifiltration: a family of spaces parameterized along multiple geometric dimensions. In this paper, we show that no similar complete discrete invariant exists for multidimensional persistence. Instead, we propose the rank invariant, a discrete invariant for the robust estimation of Betti numbers in a multifiltration, and prove its completeness in one dimension.
\end{abstract}

Keywords Computational topology $\cdot$ Multidimensional analysis $\cdot$ Persistent homology $\cdot$ Persistence

\section{Introduction}

In this paper, we introduce the theory of multidimensional persistence, an extension of the concept of persistent homology [9, 21]. Persistence captures the topology of a filtration, a one-parameter increasing family of spaces. Filtrations arise naturally from many processes, such as multiscale analysis of noisy datasets. Given a filtration,

\footnotetext{
The first author was partially supported by NSF under grant DMS-0354543. The second author was partially supported by DARPA under grant HR 0011-06-1-0038 and by ONR under grant N 00014-08-1-0908. Both authors were partially supported by DARPA under grant HR 0011-05-1-0007.

\section{G. Carlsson}

Department of Mathematics, Stanford University, Stanford, CA, USA
}

\section{A. Zomorodian $(\bowtie)$}

Department of Computer Science, Dartmouth College, Hanover, NH, USA

e-mail: afra@cs.dartmouth.edu 


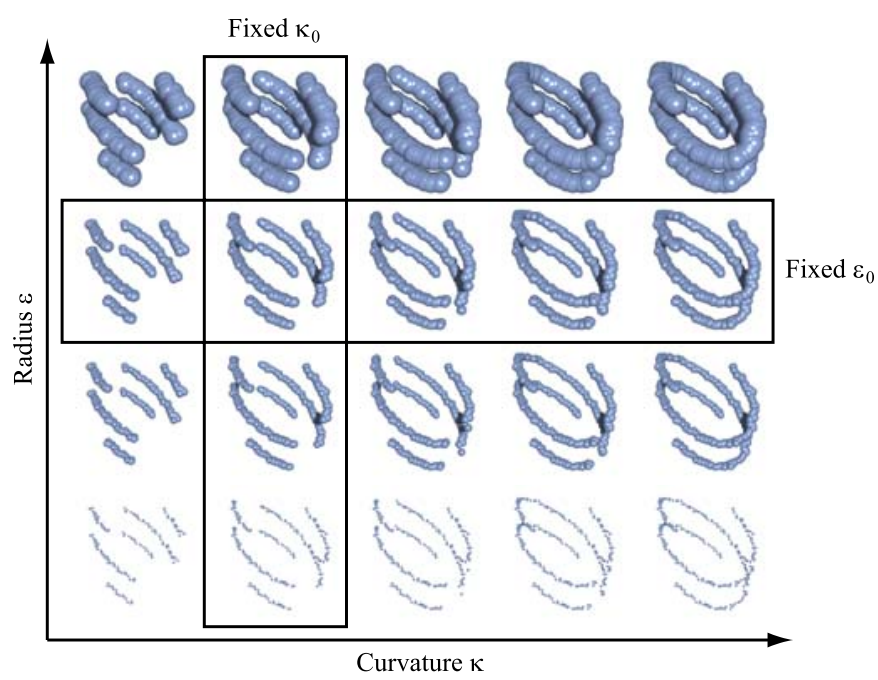

Fig. 1 A bifiltration parameterized along curvature $\kappa$ and radius $\epsilon$. We can only apply persistent homology to a filtration, so we must either fix $\epsilon$ or $\kappa$

persistent homology provides a small description in terms of a multiset of intervals we call the barcode. The intervals correspond to the lifetimes of the topological attributes. Since features have long lives, while noise is short-lived, a quick examination of the intervals enables a robust estimation of the topology of a dataset. This estimation is the key reason for the current popularity of persistent homology for solving problems in diverse disciplines, such as shape description [6], denoising volumetric density data [13], detecting holes in sensor networks [8], and analyzing the structure of natural images [3, 7]. For recent surveys, see [11, 20].

We often encounter richer structures that are described by multiple parameters. These structures may be modeled with multifiltrations, such as the bifiltration shown in Fig. 1. In previous work, we provided the theoretical foundations for the persistent homology of single parameter filtrations, obtaining a simple classification over fields in terms of the barcode [21]. Significantly, we showed that the barcode was complete, capturing all the topological information within a filtration. In this paper, we show that a similar result is unattainable for multidimensional filtrations: there exists no small complete description, like the barcode, in higher dimensions. Given this negative theoretical result, we still desire a discriminating invariant that enables detection of persistent features in a multifiltration. To this end, we propose the rank invariant. In one dimension, this invariant is equivalent to the barcode and consequently complete. Unlike the barcode, however, the rank invariant extends to higher dimensions, where it still captures persistent features, making it useful for practical applications.

\subsection{Motivation}

Filtrations arise naturally whenever we attempt to study the topological invariants of a space computationally. Often, our knowledge of a space is limited and imprecise. 
Consequently, we utilize a multiscale approach to capture the connectivity of the space, giving us a filtration.

Example 1 (Radius $\epsilon$ ) We often have a finite set of noisy samples from a subspace $X \subset \mathbb{R}^{n}$, such as the point set at the bottom of the vertical box in Fig. 1. If the sampling is dense enough, we should be able to compute the topological invariants of $X$ directly from the points [4]. To do so, we approximate the original space as a union of balls by placing $\epsilon$-balls around each point. As we increase $\epsilon$, we obtain a family of nested spaces or a filtration, as shown in the vertical box in Fig. 1.

The example sketches the basic idea behind many methods for computing the topology of a point set, such as Čech, Rips-Vietoris [12], or witness [7] complexes. On the other hand, we often study spaces that are filtered to begin with, and the filtrations contains important information that we wish to extract.

Example 2 (Density $\rho$ ) Suppose that we have a probability density function $\delta$ on $X \subset \mathbb{R}^{n}$. We can define

$$
X_{\rho}=\{x \in X \mid 1 / \delta(x) \leq \rho\} .
$$

Clearly, $X_{\rho_{1}} \subseteq X_{\rho_{2}}$ for $\rho_{1} \leq \rho_{2}$, so $\left\{X_{\rho}\right\}_{\rho}$ is a filtration. We can obtain information about $\delta$ from this filtered space. For instance, the number of persistent connected components gives an estimate of the number of the modes of $\delta$. In higher dimensions, one may uncover even more interesting structure, as was demonstrated for the nine-dimensional data set of $3 \times 3$ image patches constructed by Lee, Mumford, and Pedersen [15] and analyzed by topological methods [3, 7].

Example 3 (Curvature $\kappa$ ) In prior work [2], we develop a methodology for obtaining compact shape descriptors for manifolds by examining the topology of derived spaces. Our approach constructs the tangent complex, the closure of the tangent bundle, and filters it using curvature, as shown in the horizontal box in Fig. 1. We show that the persistence barcodes of the filtered tangent complex are useful shape descriptors.

In practice, we often have a finite set of samples from our space, giving us a filtered point set. Given a point set, we may employ the technique in Example 1 to capture topology, constructing a filtration based on increasing the radius $\epsilon$. But when the point set itself is filtered, our solution lies within the persistent homology along other geometric dimensions, such as density $\rho$ in Example 2, or curvature $\kappa$ in Example 3. We now have multiple dimensions along which our space is filtered, that is, we have a multifiltration. Of course, we could apply persistent homology along any single dimension by fixing the value of the other parameters, as indicated by the boxes in the figure [6]. However, persistent homology itself was motivated by our inability to robustly estimate values for these parameters. To eliminate the need for fixing values, we wish to apply persistence along all dimensions at once. Our goal is to be able to identify persistent features by examining the entire multifiltration. We call this problem multidimensional persistence. Variants of this problem have appeared in other contexts, such as the first size homotopy groups [10]. 


\subsection{Approach}

To understand the structure of multidimensional persistence, we utilize a general algebraic approach consisting of three steps:

1. correspondence,

2. classification, and

3. parameterization.

In the first step, we identify the algebraic structure that corresponds to our space of interest. In the second step, we obtain a complete classification of the structure, up to isomorphism. In the third step, we parameterize the classification. Our parameterization will be in the form of invariants. An invariant is a function on a set of structures (e.g., modules, graded modules, etc.) which takes values in another (typically explicitly describable) set, called the parameter set, and whose values on isomorphic structures are identical. An example is the trivial invariant, which assigns the same element within a one element parameter set to all structures and is therefore useless. A complete invariant, on the other hand, always assigns different elements in the parameter space to structures that are not isomorphic. Complete invariants are the most powerful type of invariant, and we naturally search for them. If complete invariants do not exist, we search for incomplete invariants that have enough discriminating power to be useful.

Our goal is to obtain a useful parameterization consisting of a small set of invariants whose description is finite in size. We utilize terminology from algebraic geometry to distinguish between invariants. We seek invariants that correspond to points in algebraic varieties and do not depend on the underlying field of computation. The former condition enables them to have finite parameterizations. The latter means that our invariant always comes from the same set, similar to the Betti numbers, which are always integers regardless of the coefficient ring. For brevity, we call these invariants discrete, and other invariants continuous. Continuous invariants may be uncountable in size or depend on the underlying field of computation. Naturally, these invariants are not viable from a computational point of view. Therefore, our objective is a complete discrete invariant for multidimensional persistence. We note that our notation has nothing to do with whether the underlying field of computation is continuous, such as $\mathbb{R}$, or discrete, such as $\mathbb{F}_{p}$ for a prime $p$.

\subsection{One-Dimensional Persistence}

In a previous paper, we follow the algebraic approach enumerated above and obtain a complete discrete invariant for one-dimensional persistence [21]:

1. Correspondence: We show a correspondence between the homology of a filtration in any dimension and a graded $R[t]$-module, where $R[t]$ is the ring of polynomials with indeterminate $t$ over ring $R$.

2. Classification: Over fields $k, k[t]$ is a principal ideal domain, so a consequence of the standard structure theorem for graded $k[t]$-modules gives the full classifica- 
tion:

$$
\bigoplus_{i=1}^{n} \Sigma^{\alpha_{i}} k[t] \oplus \bigoplus_{j=1}^{m} \Sigma^{\gamma_{j}} k[t] /\left(t^{n_{j}}\right),
$$

where $\Sigma^{\alpha}$ denotes an $\alpha$-shift upward in grading.

3. Parameterization: The classification gives us $n$ half-infinite intervals $\left[\alpha^{i}, \infty\right)$ and $m$ finite intervals $\left[\gamma_{j}, \gamma_{j}+n_{j}\right)$. The multiset of $n+m$ intervals is a complete discrete invariant. We call this multiset the persistence barcode [2].

This description shows that we have achieved our stated objective completely for the case $n=1$.

\subsection{Contributions}

In this paper, we show that multidimensional persistence has an essentially different character from its one-dimensional version. We devote a major portion of this paper to the following theoretical contributions:

- We identify the algebraic structure that corresponds to multidimensional persistence to be a finitely-generated multigraded module over the field of multivariate polynomials.

- We establish a full classification of this structure in terms of the set of the orbits of the action of an algebraic group on an algebraic variety.

- We reveal that this classification has discrete and continuous portions. The former is canonically parameterizable, but the latter has no precise parameterization.

Our results imply that no complete discrete invariant exists for multidimensional persistence, unlike its one-dimensional counterpart. Given this negative result, we conclude the paper by describing a practical invariant:

- We propose a discrete invariant, the rank invariant, that is computable, compact, and useful for extracting persistence information from multifiltrations.

- We prove the rank invariant is equivalent to the persistence barcode in one dimension, making it complete for one-dimensional persistence, the only type for which it can be complete.

Our work has both theoretical and practical components, the former being a full understanding of multidimensional persistence, and the latter being a practical invariant that is useful for computation. In Sect. 2, we review concepts from algebra, algebraic topology, and algebraic geometry and invent some notation. The next three sections detail the three steps of our approach, respectively. In Sect. 6, we propose our discrete invariant for multidimensional persistence and show its completeness in one dimension.

\section{Background}

Let $\mathbb{N}$ be the set of nonnegative integers, also called the natural numbers. For $u, v \in \mathbb{N}^{n}$, we say $u \lesssim v$ if $u_{i} \leq v_{i}$ for $1 \leq i \leq n$. A multiset is a set within which 
an element may appear multiple times, such as $\{a, a, b, c\}$. We will formalize this notion in Sect. 4.1. A monomial in $x_{1}, \ldots, x_{n}$ is a product of the form

$$
x_{1}^{v_{1}} \cdot x_{2}^{v_{2}} \cdots x_{n}^{v_{n}}
$$

with $v_{i} \in \mathbb{N}$. We denote it $x^{v}$, where $v=\left(v_{1}, \ldots, v_{n}\right) \in \mathbb{N}^{n}$. A polynomial $f$ in $x_{1}, \ldots, x_{n}$ and coefficients in field $k$ is a finite linear combination of monomials, $f=\sum_{v} c_{v} x^{v}$, with $c_{v} \in k$. We denote the set of all polynomials $k\left[x_{1}, \ldots, x_{n}\right]$. For example, $5 x_{1} x_{2}^{2}-7 x_{1}^{3} \in \mathbb{R}\left[x_{1}, x_{2}\right]$ has two nonzero coefficients: $c_{(1,2)}=5$ and $c_{(3,0)}=-7$.

An algebraic variety is the set of common zeros of a collection of polynomials. One variety we encounter in this paper is the Grassmannian $\mathrm{Gr}_{k}(V)$, the set of $k$-dimensional subspaces of a vector space $V$. An algebraic group is an algebraic variety endowed with group structure, so that the group operation is a morphism of the variety. An automorphism is an isomorphism of a mathematical object to itself. The set of all automorphisms of a set of objects $V$ forms the automorphism group $\operatorname{Aut}(V)$. When the objects $V$ are vector spaces, the automorphism group is the general linear group $\mathrm{GL}(V)$, the set of invertible linear transformations on $V$, where the group operation is function composition.

Let $S$ be a set and $G$ be a group. An action of $G$ on $S$ is a binary operation $*: G \times S \rightarrow S$ such that for the identity element $e \in G$, we have $e * s=s$ for all $s \in S$, and $\left(g_{1} g_{2}\right) * s=g_{1} *\left(g_{2} * s\right)$ for all $s \in S$ and $g_{1}, g_{2} \in G$. Given a group action, we define $s_{1} \sim s_{2}$ iff there exists $g \in G$ such that $g * s_{1}=s_{2}$. Then, $\sim$ is an equivalence relation on $S$ and partitions it. Each cell in the partition is an orbit in $S$ under $G$.

An $n$-graded ring is a ring $R$ equipped with a decomposition of Abelian groups $R \cong \bigoplus_{v} R_{v}, v \in \mathbb{N}^{n}$, so that multiplication has the property $R_{u} \cdot R_{v} \subseteq R_{u+v}$. The set of polynomials $A_{n}=k\left[x_{1}, \ldots, x_{n}\right]$ forms the polynomial ring. $A_{n}$ is graded by $A_{v}=k x^{v}, v \in \mathbb{N}^{n}$, and is the prototype for $n$-graded rings. We may visualize the 2-graded ring $A_{2}$ on the integer grid $\mathbb{N}^{2}$, as shown in Fig. 3(a), where each bullet is a grade that contains an element from $k$. Our example polynomial $5 x_{1} x_{2}^{2}-7 x_{1}^{3}$ has nonzero elements in grades $(1,2)$ and $(3,0)$. An $n$-graded module over an $n$-graded $\operatorname{ring} R$ is an Abelian group $M$ equipped with a decomposition $M \cong \bigoplus_{v} M_{v}, v \in \mathbb{N}^{n}$, together with a $R$-module structure so that $R_{u} \cdot M_{v} \subseteq M_{u+v}$.

\section{Correspondence}

In this section, we carry out the first step of the approach outlined in Sect. 1.2: identifying the algebraic structure underlying our problem. The abstraction for our input is a multifiltered space. A space $X$ is multifiltered if we are given a family of subspaces $\left\{X_{v} \subseteq X\right\}_{v \in \mathbb{N}^{n}}$ with inclusions $X_{u} \subseteq X_{w}$ whenever $u \lesssim w$, so that the diagrams

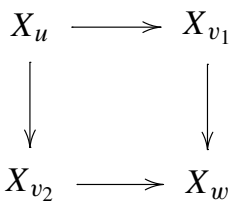

commute for $u \lesssim v_{1}, v_{2} \lesssim w$. We showed an example of a bifiltration in Fig. 1 . 
Fig. 2 A bifiltration of a triangle

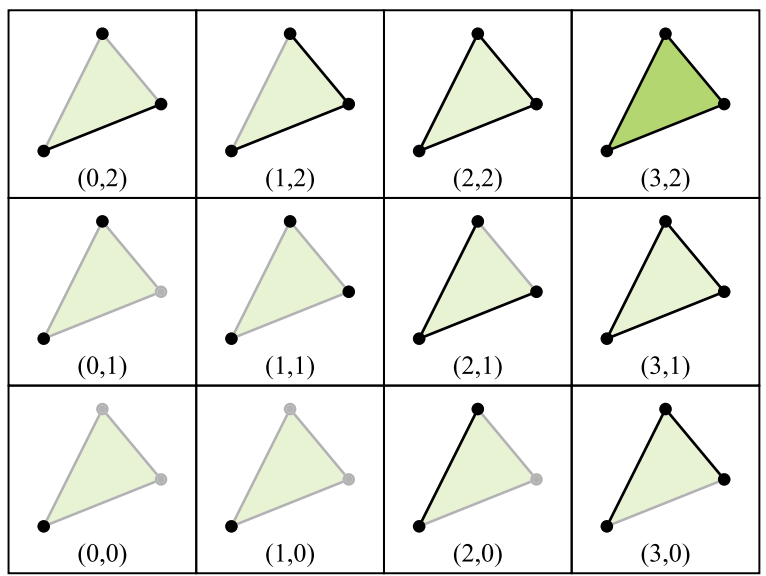

In practice, our input is often a finite complex $K$ along with a function $F$ : $\mathbb{R}^{n} \rightarrow K$ that gives a subcomplex $K_{v}$ for any value $v \in \mathbb{R}^{n}$, such as the bifiltered triangle in Fig. 2. This input converts naturally to a multifiltered complex. Since the complex is finite, there is a finite set of critical coordinates $C=\left\{v_{i} \in \mathbb{R}^{n}\right\}_{i}$ at which new simplices enter the complex. Projecting $C$ onto each coordinate axis gives us a finite set of critical values $C_{d}$ in each dimension $d$. We now restrict ourselves to the discrete set of the Cartesian product $\prod_{d=1}^{n} C_{d}$ of the critical values, parameterizing the resulting grid using $\mathbb{N}$ in each dimension. This gives us a multifiltered complex, provided that the function $F$ makes the induced diagrams (1) commute.

Given a multifiltered space $X$, the homology of each subspace $X_{v}$ over a field $k$ is a vector space. For instance, the bifiltered complex in Fig. 2 has zeroth homology vector spaces isomorphic to the commutative diagram

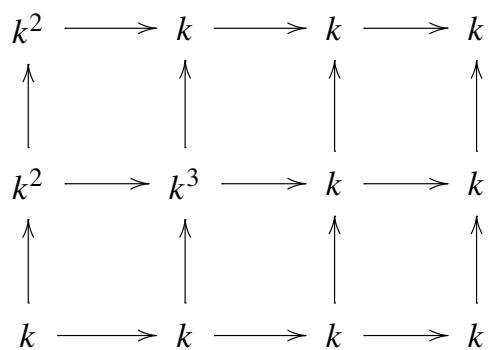

where the dimension of the vector space counts the number of components of the complex, and the maps between the homology vector spaces are induced by the inclusion maps relating the subspaces.

Definition 1 (Persistence module) A persistence module $M$ is a family of $k$-modules $\left\{M_{v}\right\}_{v}$ together with homomorphisms $\varphi_{u, v}: M_{u} \rightarrow M_{v}$ for all $u \lesssim v$ such that $\varphi_{u, v}$ 。 $\varphi_{v, w}=\varphi_{u, w}$ whenever $u \lesssim v \lesssim w$. 
The homology of a multifiltration in each dimension is a persistence module. To capture the structure of the maps in a persistence module, we define a multigraded module, following our treatment in the one-dimensional case [21].

Definition 2 (Structure) Given a persistence module $M$, we define an $n$-graded module over $A_{n}$ by

$$
\alpha(M)=\bigoplus_{v} M_{v}
$$

where the $k$-module structure is the direct sum structure, and we require that $x^{v-u}: M_{u} \rightarrow M_{v}$ is $\varphi_{u, v}$ whenever $u \lesssim v$.

That is, we incorporate the relationships given by the homomorphisms into the structure of an $n$-graded module. Our treatment is consistent with, and an extension of, the one-dimensional case, where the corresponding structure is a 1-graded or singly-graded module [21].

Theorem 1 (Correspondence) The correspondence $\alpha$ defines an equivalence of categories between the category of finite persistence modules over $k$ and the category of finitely generated $n$-graded modules over $A_{n}=k\left[x_{1}, \ldots, x_{n}\right]$.

To recap, the homology of a finite multifiltered complex is a finite persistence module, and the structure of a persistence module is a finitely generated $n$-graded module.

One may ask about the reverse relationship: Is every finite persistence module realizable as the homology of a multifiltration? More specifically, can we realize every such module as the homology of a finite multifiltered simplicial complex, since that is our usual representation of a space in practice? The following theorem answers this question in the affirmative.

Theorem 2 (Realization) Let $k=\mathbb{F}_{p}$ for some prime $p$, let l be a positive integer, and let $M$ be an $n$-graded module over $k\left[x_{1}, \ldots, x_{n}\right]$. Then there is a multifiltered finite simplicial complex $X$ so that $H_{k}(X, k) \cong M$ as persistence modules.

The proof is constructive, and we omit it here.

We end this section with an aside on our choice of input. The grid-like filtrations that we study arise naturally in practice. Nevertheless, filtrations arising from other partial orders may also be interesting and produce algebraic invariants. However, this would take us out of the realm of commutative algebra, perhaps into noncommutative algebra, and definitely into another paper.

\section{Classification}

We have now identified the algebraic structures which correspond to our problem: finitely generated $n$-graded modules over $A_{n}$. In this section, we focus on our second 
task: finding a complete classification up to isomorphism of these objects. The general idea is to observe that the first two stages of a minimal free resolution of $M$ are unique up to isomorphism of free chain complexes.

\subsection{Multigraded Sets and Multisets}

Let $n$ be a positive integer. By an $n$-graded set, we will mean a pair $(X, \varphi)$, where $X$ is a set, and $\varphi: X \rightarrow \mathbb{Z}^{n}$ is a map of sets. A map of graded sets is of course a set map compatible with the reference maps $\varphi$ in the obvious way. For any $n$-graded $A_{n}$-module $M$, we associate to it an $n$-graded set $\mathbb{H}(M)=\bigcup_{v \in \mathbb{Z}^{n}} M_{v}$, the homogeneous elements in $M$. We will find it useful to think of graded sets in terms of multisets. A multiset is a subset $L$ of $\mathbb{Z}^{n}$, together with a map $\alpha: L \rightarrow \mathbb{N}$, where $\mathbb{N}$ denotes the natural numbers $\{1,2, \ldots, n, \ldots\}$. We note that the set $\mathbb{Z}^{n} \times \mathbb{N}$ can be given the structure of an $n$-graded set via the projection map $\mathbb{Z}^{n} \times \mathbb{N} \rightarrow \mathbb{Z}^{n}$. A multiset $(L, \alpha)$ now specifies a graded subset of $\mathbb{Z}^{n} \times \mathbb{N}$ given by $\{(v, n) \mid n \leq \alpha(v)\}$. This will be a convenient way of thinking about $n$-graded sets.

Let $(S, \mu)$ be any multiset where $S \subseteq \mathbb{N}^{n}$. Then, the relation $\lesssim$ is a quasi-partial order on $(S, \mu)$ : It is reflexive and transitive, but not anti-symmetric, since elements appear with multiplicity.

\subsection{Free Graded Objects}

Let $B$ be any $k$-algebra, where $k$ is a field. The usual notions of the free $B$-module on a set or on a $k$-vector space admit generalizations to the multigraded setting. By a free $A_{n}$-module on the graded set $(X, \varphi)$ we will mean an $n$-graded $A_{n}$-module $F$, together with an inclusion of $n$-graded sets

$$
\eta:(X, \varphi) \hookrightarrow \mathbb{H}(F) \subseteq F,
$$

so that for any $n$-graded $A_{n}$-module $M$ and map of $n$-graded sets $\theta:(X, \varphi) \rightarrow \mathbb{H}(M)$, there is a unique homomorphism $\lambda: F \rightarrow M$ of $n$-graded $A_{n}$-modules so that the diagram

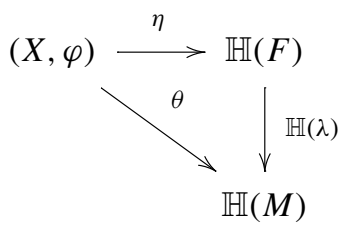

commutes, where $\mathbb{H}(\lambda)$ denotes the restriction of $\lambda$ to the homogeneous elements. Note that any homomorphism of $n$-graded modules preserves homogeneous elements. It is routine to check that such modules exist, and the hypotheses guarantee that they are unique up to isomorphism. A particular example of this construction is that of a free $n$-graded vector space. In this case, it is easy to show that all $n$-graded vector spaces are free, by analogy with the ungraded case. For any $n$-graded $A_{n}$-module $M$, we construct a new $n$-graded module $M(v)$ for any $v \in \mathbb{Z}^{n}$ by setting $M(v)_{w}=M_{w-v}$. The module structure follows directly, and this module is thought 


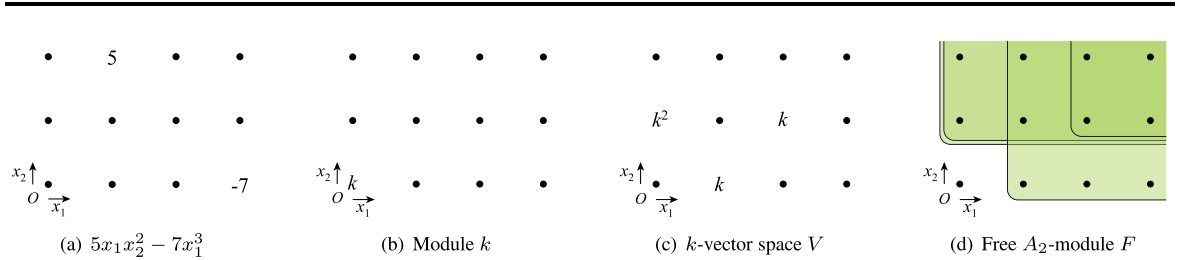

Fig. 3 2-graded objects: (a) Polynomial $5 x_{1} x_{2}^{2}-7 x_{1}^{3}$ in the 2-graded ring $A_{2}=\mathbb{R}\left[x_{1}\right.$, $\left.x_{2}\right]$ visualized on $\mathbb{N}^{2}$. (b) Field $k$ endowed with graded module structure. (c) A $k$-vector space $V$ with generators at $(1,0)$ and $(2,1)$, and two generators at $(0,1)$. (d) A free $A_{2}$-module $F$ with same type as (b)

of as obtained by shifting the grading by $v$. Any finitely generated free $n$-graded module can be expressed as $\bigoplus_{i} A_{n}\left(v_{i}\right)$ for some family of $v_{i}$ 's. Similarly, every finite-dimensional $n$-graded vector space, such as the vector space in Fig. 3(c), can be described as $\bigoplus_{i} k\left(v_{i}\right)$ for some choice of $v_{i}$ 's, where $k$ denotes the ground field regarded as an $n$-graded module $k$ where $k_{v}=\{0\}$ for $v \neq \overrightarrow{0}$, and $k_{0} \cong k$, as shown in Fig. 3(b). Finally, one can check that if we are given two $n$-graded sets $(X, \varphi)$ and $\left(X^{\prime}, \varphi^{\prime}\right)$ such that the free $n$-graded module (or free $n$-graded $k$-vector space) on $X$ is isomorphic to the corresponding construction for $X^{\prime}$, then the two graded sets $(X, \varphi)$ and $\left(X^{\prime}, \varphi^{\prime}\right)$ are also isomorphic. In other words, the graded bases for the modules are isomorphic as graded sets. We use this fact to define the type of an $n$-graded vector space $V$ as the unique multiset which is isomorphic to a graded basis for $V$, and denote it $\xi(V)$. Similarly, we define $\xi(F)$ for any free $n$-graded $A_{n}$-module.

Finally, we note that for any $n$-graded $k$-vector space $V$, there is free $n$-graded $A_{n}$-module on $V$. It satisfies the obvious universality property, and we will denote it by $F(V)$. As in the usual case, there is a canonical construction, namely $A_{n} \otimes_{k} V$, as shown in Fig. 3(d).

\subsection{Automorphisms}

We wish to analyze the automorphism group of a free $n$-graded module $F$. For any multiset $\xi$, we will denote by $V(\xi)$ and $F(\xi)$ a particular choice of a free $n$-graded $k$-vector space and an $n$-graded $A_{n}$-module, respectively. Note that any two such are canonically isomorphic, in that there is a unique basis preserving map from the one to the other. We first analyze the automorphism group of an $n$-graded $k$-vector space.

Theorem 3 Let $V$ be an $n$-graded vector space over $k$, of type

$$
\xi=\left\{\left(v_{1}, n_{1}\right),\left(v_{2}, n_{2}\right), \ldots,\left(v_{s}, n_{s}\right)\right\}
$$

where all the $v_{i}$ 's are distinct. Then the automorphism group is isomorphic to the product $\prod_{i} \mathrm{GL}_{n_{i}}(k)$.

Proof $V$ is isomorphic to the direct sum $\bigoplus_{i} V_{v_{i}}$, and by the definitions, any automorphism of $V$ must preserve this direct sum decomposition. Consequently, $\operatorname{Aut}(V) \cong$ $\prod \operatorname{Aut}\left(V_{v_{i}}\right)$ and $V_{v_{i}} \cong k^{n_{i}}$, giving the result. 
The situation of an $n$-graded $A_{n}$-module is a bit more subtle, since in this case the ring has elements in degrees other than 0 . We describe the structure of $\operatorname{Aut}(F(\xi))$ as an algebraic group over $k$. In order to do this, we will need to make some definitions.

Definition 3 Let $n$ be a positive integer. By an $n$-multifiltered $k$-vector space, we will mean a $k$-vector space $V$, together with a family $\mathcal{F}$ of subspaces $\mathcal{F}_{w} V$ for every $w \in \mathbb{Z}^{n}$, so that:

1. If $w \leq w^{\prime}$, then $\mathcal{F}_{w} V \subseteq \mathcal{F}_{w^{\prime}} V$.

2. There is a $w_{0} \in \mathbb{Z}^{n}$ such that $\mathcal{F}_{w_{0}} V=V$.

3. There is a $w_{1} \in \mathbb{Z}^{n}$ such that $\mathcal{F}_{w_{1}} V=\{0\}$.

For any $n$-graded vector space $V$, we let $\mathcal{F} V$ denote the associated $n$-multifiltered vector space to have underlying vector space $V$, and, for every $w \in \mathbb{Z}^{n}$, we set

$$
\mathcal{F}_{w} V=\bigoplus_{w^{\prime} \leq w} V_{w^{\prime}}
$$

It is clear what is meant by a morphism of $n$-multifiltered vector spaces, namely a homomorphism of underlying vector spaces which respects the subspaces corresponding to $w$ for each $w \in \mathbb{Z}^{n}$. It is also clear that the correspondence $\mathcal{F}$ is a functor, indeed that a morphism of the $n$-multigraded structure can be viewed directly as a morphism of the associated multifiltered objects.

Theorem 4 For any finite-dimensional $n$-multifiltered vector space $(V, \mathcal{F})$, the automorphism group of $(V, \mathcal{F})$ is an algebraic subgroup of the full automorphism group $\mathrm{GL}(V)$ of the underlying vector space $V$. For any finite dimensional $n$-graded vector space $V, \operatorname{Aut}(V)$ is an algebraic subgroup of the algebraic group $\operatorname{Aut}(\mathcal{F} V)$.

Proof Straightforward verification.

Let $M$ denote any finitely generated $n$-graded $A_{n}$-module. We define the finitedimensional $k$-vector space $\rho(M)=k \otimes_{A_{n}} M$, where $k$ is given the module structure where all the variables $x_{i}$ act trivially, i.e., by zero. We now have the following:

Theorem 5 (Automorphisms) For any finitely generated free $n$-graded $A_{n}$-module $F$, there is an isomorphism

$$
\operatorname{Aut}(F) \cong \operatorname{Aut}(\mathcal{F}(\rho(F)))
$$

where the second automorphism group is computed in the category of n-multifiltered $k$-vector spaces. Consequently, Aut $(F)$ has the structure of an algebraic group in a natural way.

Proof We write $F \cong \bigoplus_{i} A\left(v_{i}\right)$ for some finite set of vectors $\left\{v_{i}\right\}$. We write $e_{i}$ for the basis element corresponding to the $i$ th factor, so $e_{i} \in F_{v_{i}}$. We now have $\rho(F) \cong$ $\bigoplus_{i} k\left(v_{i}\right)$, and a basis for $\rho(F)$ is given by the elements $\overline{e_{i}}$, which are the reductions 
of $e_{i}$ in $\rho(F)$. Given these basis elements, any automorphism $\varphi$ of the $n$-multifiltered $k$-vector space $\rho(F)$ is determined by a choice of elements $\alpha_{i j} \in k$, so that

$$
\varphi\left(\overline{e_{j}}\right)=\sum_{i} \alpha_{i j} \overline{e_{i}}
$$

and $\alpha_{i j}=0$ whenever $v_{j} \ngtr v_{i}$. The $\alpha_{i j}$ 's also determine an automorphism $\tilde{\varphi}$ of $F$ itself via the formula

$$
\tilde{\varphi}\left(e_{j}\right)=\sum_{i} \alpha_{i j} x^{v_{j}-v_{i}} e_{i} .
$$

It is clear that the correspondence $\varphi \rightarrow \tilde{\varphi}$ is actually a homomorphism from $\operatorname{Aut}(\mathcal{F} \rho(F))$ to $\operatorname{Aut}(F)$. There is a homomorphism in the reverse directions which carries an automorphism $\psi$ of $F$ to the automorphism $i d_{k} \otimes_{A_{n}} \psi$ of $\rho(F)$, and it is clear that these are inverse correspondences.

\subsection{Free Hulls}

We will wish to interpret isomorphism classes of modules in terms of isomorphism classes of two stages complexes which extend to a resolution of the given module. In order to relate modules with resolutions, we state the following elementary extension of an easy consequence of Nakayama's Lemma [1]. The theorem is the extension to the $n$-graded cases of the similar theorem for graded modules over graded rings [17].

Theorem 6 Let $f: M \rightarrow N$ be a homomorphism of finitely generated free $n$-graded $A_{n}$-modules, and suppose that

$$
i d_{k} \otimes_{A_{n}} f: k \otimes_{A_{n}} M \rightarrow k \otimes_{A_{n}} N
$$

is an isomorphism of $n$-graded $k$-vector spaces. Then $f$ is an isomorphism.

Definition 4 For a finitely generated $n$-graded $A_{n}$-module $M$, a free hull for $M$ will be any surjective homomorphism $p: F \rightarrow M$ of $n$-graded modules, where $F$ is a finitely generated free $n$-graded $A_{n}$-module, and such that the induced map

$$
i d_{k} \otimes_{A} p: k \otimes_{A} F \rightarrow k \otimes_{A} M
$$

is an isomorphism.

Theorem 7 Every finitely generated $n$-graded $A_{n}$-module admits a free hull. Moreover, any two free hulls for $M$ are isomorphic in the sense that if $p: F \rightarrow M$ and $p^{\prime}: F^{\prime} \rightarrow M$ are both free hulls, then there is a commutative diagram

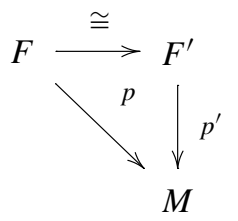

where the horizontal arrow is an isomorphism. 
Proof This is standard in the ordinary graded (or local ring) situation, and the proof here is identical, using Theorem 6 in place of the standard result from the theory of local rings.

\subsection{Complete Classification}

We begin by defining two multiset-valued invariants of finitely generated $n$-graded $A_{n}$-modules $M$. We define the invariant $\xi_{0}(M)$ to be $\xi(\rho(M))$, i.e., the type of the $n$-graded $k$-vector space $\rho(M)$. This is clearly an invariant of the isomorphism class of the module. For the second invariant, select any free hull $p: F \rightarrow M$ of $M$, and let $K \subseteq F$ denote the kernel of $p$. Then we define $\xi_{1}(M)$ to be $\xi(\rho(K))$.

Theorem 8 The multiset $\xi_{1}(M)$ is independent of the free hull chosen, hence is a multiset-valued invariant of the isomorphism class of $M$.

Proof Given any two free hulls $p: F \rightarrow M$ and $p^{\prime}: F^{\prime} \rightarrow M$, Theorem 7 asserts that there is an isomorphism $\theta: M \rightarrow M^{\prime}$, so that $p^{\prime} \circ \theta=p$. It is immediate that $\theta$ restricts to an isomorphism from $K$ to $K^{\prime}=\operatorname{ker}\left(p^{\prime}\right)$. Since $K$ and $K^{\prime}$ are isomorphic, $\xi(\rho(K))=\xi\left(\rho\left(K^{\prime}\right)\right)$, which gives the result.

Suppose now that we are given two multisets $\xi_{0}$ and $\xi_{1}$. We first construct a free $n$-graded $A_{n}$-module $F$ so that $\xi(\rho(F))=\xi_{0}$. Next, we let $\mathcal{S}\left(F, \xi_{1}\right)$ denote the set of all $n$-graded $A_{n}$-submodules $L$ of $F$ for which $\xi(\rho(L))=\xi_{1}$. Note that the automorphism group of $F$ acts on $\mathcal{S}\left(F, \xi_{1}\right)$ via $g \cdot K=g(K)$ for $g \in \operatorname{Aut}(F)$. Next, let $\mathcal{I}\left(\xi_{0}, \xi_{1}\right)$ denote the set of isomorphism classes of $n$-graded $A_{n}$-modules $M$ for which $\xi_{0}(M)=\xi_{0}$ and $\xi_{1}(M)=\xi_{1}$. There is a map $q: \mathcal{S}\left(F, \xi_{1}\right) \rightarrow \mathcal{I}\left(\xi_{0}, \xi_{1}\right)$ defined by

$$
q(K)=[F / K]
$$

where $[-]$ denotes isomorphism class.

Theorem 9 (Classification) Let $F$ be as above, and denote $\operatorname{Aut}(F)$ by $G_{F}$. The map $q$ satisfies the formula $q(g \cdot K)=q(K)$ and consequently induces a map $\bar{q}: G_{F} \backslash \mathcal{S}\left(F, \xi_{1}\right) \rightarrow \mathcal{I}\left(\xi_{0}, \xi_{1}\right)$. Moreover, $\bar{q}$ is a bijection.

Proof For any $g \in G_{F}$, we see immediately that action by $g$ carries $K$ into $g(K)$, and therefore we obtain an induced isomorphism $g: F / K \rightarrow F / g(K)$. This shows that $q(K)=q(g \cdot K)$. To see that $\bar{q}$ is surjective, it suffices to prove the same result for $q$. But now, Theorem 7 shows that there exists a surjection from $\pi: F \rightarrow M$ if $\xi(\rho(M))=\xi_{0}$. This relies on the observation that there is a unique (up to isomorphism) free $n$-graded $A_{n}$-module $F$ with $\xi(\rho(F))=\xi_{0}$. Now $\operatorname{ker}(\pi)$ is now an element in $\mathcal{S}(F)$, and clearly $q(\operatorname{ker}(\pi))=[M]$, demonstrating surjectivity. For injectivity, we suppose that we are given $K, K^{\prime} \in \mathcal{S}(F)$ and that $q(K)=q\left(K^{\prime}\right)$, i.e., $F / K \cong F / K^{\prime}$. Let $\alpha: F / K \rightarrow F / K^{\prime}$ be an isomorphism. Theorem 7 now shows that 
there is an automorphism $\tilde{\alpha}$ of $F$ such that the diagram

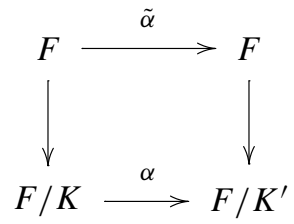

commutes. It is clear that $\alpha$ carries $K$ isomorphically to $K^{\prime}$, which shows that $K$ and $K^{\prime}$ represent the same orbit in $\mathcal{I}\left(\xi_{0}, \xi_{1}\right)$.

\section{Parameterization}

Having established a complete classification of the graded modules, we now turn our attention to the third step of our approach: parameterizing the classification. We have shown earlier in Theorem 5 that $\operatorname{Aut}(F)$ is an algebraic group. We now show that the elements of $\mathcal{S}\left(\xi_{0}, \xi_{1}\right)$ are naturally identified with the points of an algebraic variety, and further that the action of $\operatorname{Aut}(F)$ on them is an algebraic group action. The general picture that emerges is that this portion of the classification is a continuous invariant. To appreciate its nature, we next detail an example in two dimensions. We end this section with possible strategies for coping with the continuous invariant.

\subsection{Interpretation via Algebraic Varieties}

We begin by considering any $n$-graded $A_{n}$-module $M$. For every $v \in \mathbb{Z}^{n}$, we consider the $k$-vector subspace

$$
(I M)_{v}=\sum x_{i} M_{v-e_{i}} \subseteq M_{v},
$$

where $e_{i}$ denotes the $i$ th standard basis vector in $\mathbb{Z}^{n}$. We say $v$ is a gap for $M$ if $(I M)_{v} \neq M_{v}$. Let $\Gamma(M)$ denote the set of gaps of $M$.

Theorem 10 If $M$ is finitely generated, then $\Gamma(M)$ is finite. Moreover, the type of $\rho(M), \xi(\rho(M))$, is the multiset $\left(\Gamma(M), \alpha_{M}\right)$, where

$$
\alpha_{M}(v)=\operatorname{dim}_{k}\left(M_{v} /(I M)_{v}\right)=\operatorname{dim}_{k}\left(M_{v}\right)-\operatorname{dim}_{k}\left((I M)_{v}\right) .
$$

Proof It is clear that the set of gaps is contained in the set of those $v$ which contain an element of the generating set, which verifies the first assertion. It is immediate from the definitions that $\rho(M)_{v} \cong M_{v} /(I M)_{v}$. It follows that the gaps are exactly those $v$ 's for which $\rho(M)_{v} \neq 0$. Further, the type of $\rho(M)$ is then clearly given by the function $\alpha_{M}$ defined above.

Theorem 11 Let $F$ denote a free $n$-graded $A_{n}$-module, and let $L$ and $L^{\prime}$ denote any $n$-graded submodule (note that unlike the case $n=1, L$ need not be free). Then $L=L^{\prime}$ if and only if the gaps of $L$ and $L^{\prime}$ are the same, and we have $L_{v}=L_{v}^{\prime}$ for all $v$ which are gaps of either. 
Proof Let $w \in \mathbb{Z}^{n}$ denote a minimal element in the set $\left\{v \in \mathbb{Z}^{n} \mid L_{v} \neq L_{v}^{\prime}\right\}$. The element $w$ must be a gap for both $L$ and $L^{\prime}$, since otherwise we find that

$$
L_{w}=(I L)_{w}=\left(I L^{\prime}\right)_{w}=L_{w}^{\prime},
$$

and the equality $(I L)_{w}=\left(I L^{\prime}\right)_{w}$ holds due to the minimality of $w$.

Let $\xi=(V, \alpha)$ denote any multiset, and let $\delta: V \rightarrow \mathbb{Z}$ be any function. For any finitely generated free $n$-graded $A_{n}$-module $F$, let $\mathbf{A R R}_{\xi, \delta}(F)$ denote the set of all assignments $v \rightarrow L_{v}$, where $v \in V$, and $L_{v}$ is a $k$-linear subspace of $F_{v}$, which satisfy the following three conditions:

1. $v^{\prime} \leq v \Longrightarrow x^{v-v^{\prime}} L_{v^{\prime}} \subseteq L_{v}$.

2. $\operatorname{dim}_{k}\left(L_{v}\right)=\delta(v)$.

3. $\operatorname{dim}_{k}\left(L_{v} / \sum_{v^{\prime}<v} x^{v-v^{\prime}} L_{v^{\prime}}\right)=\alpha(v)$ for all $v \in V$.

The goal now is to demonstrate that $\operatorname{ARR}_{\xi, \delta}(F)$ is in bijective correspondence with the set of points of a quasi-projective variety over the field $k$.

We note that algebraic geometry began with the study of affine varieties, i.e., sets of solutions of equations in affine space $k^{n}$. It was soon discovered, though, that it was extremely useful to embed the affine space into a projective space, since the statements of many theorems required fewer special cases in this context. It was also discovered that all projective varieties, over any field, are equipped with a topology, the Zariski topology. A quasi-projective variety is simply an open subset of a projective variety in this topology. Any affine variety is quasi-projective, but so would be, for example, a projective space with a single point removed.

We begin by noting that $\mathbf{A} \mathbf{R} \mathbf{R}_{\xi, \delta}(F)$ can be identified with a subset of

$$
\mathcal{E} \stackrel{\text { defn }}{=} \prod_{v \in V} \operatorname{Gr}_{\delta(v)}\left(F_{v}\right),
$$

where $\mathrm{Gr}_{k}(V)$ the Grassmann variety of $k$-dimensional subspaces of $V$, since an element of $\mathbf{A R R}_{\xi, \delta}(F)$ corresponds to a family of subspaces of dimensions determined by $\delta$, satisfying certain additional constraints. By a $(\xi, \delta)$-frame for $F$ (or frame for $F$ for short), we will mean a family of linear embeddings $\left\{j_{v}: W_{v} \rightarrow F_{v}\right\}_{v \in V}$, where $W_{v}$ is a vector space equipped with an isomorphism to $k^{\delta(v)}$. The frame determines a family of subspaces $L_{v}=\operatorname{im}\left(j_{v}\right)$. We will denote the set of $(\xi, \delta)$-frames in $F$ by $\mathcal{F}(F)$. We let $\mathrm{GL}\left(W_{v}\right)$ denote the automorphism group of the vector space $W_{v} \cong k^{\delta(v)}$. The explicit choice of isomorphism with $k^{\delta(v)}$ yields an isomorphism $\mathrm{GL}\left(W_{v}\right) \cong \mathrm{GL}_{\delta(v)}(k)$. Then, the group

$$
\Gamma=\prod_{v \in V} \mathrm{GL}\left(W_{v}\right) \cong \prod_{v \in V} \mathrm{GL}_{\delta(v)}(k)
$$

acts on $\mathcal{F}(F)$ in such a way that the elements $\left\{\sigma_{v}\right\}_{v \in V}$, with $\sigma_{v} \in \mathrm{GL}\left(W_{v}\right)$, acting on the frame $\left\{j_{v}\right\}$, yield the frame $\left\{j_{v} \circ \sigma_{v}\right\}$. The orbit space of this action is the set of all families of subspaces $\left\{L_{v}\right\}_{v \in V}$ such that $\operatorname{dim}_{k}\left(L_{v}\right)=\delta(v)$ for all $v \in V$, i.e., the set $\mathcal{E}$ defined above. 
Frames can also be thought of as matrices, as follows. We suppose that the free multigraded module $F$ is given by

$$
F \cong \bigoplus_{i=1}^{N} A_{n}\left(v_{i}\right)
$$

Select any vector $v^{*} \in \mathbb{Z}^{n}$ so that $v^{*} \geq v_{i}$ for all $1 \leq i \leq k$ and so that $v^{*}$ is greater than any element in $V$. Then each of the $k$-vector spaces $F_{v}$ is identified with a subspace $G_{v}$ of $F_{v^{*}}$ via multiplication by the monomial $x^{v^{*}-v}$, and $L_{v}$ is identified with a subspace $L_{v}^{*}$ of $G_{v}$. For each $i$ with $1 \leq i \leq k$ we let $e_{i}$ denote the generator for the summand $A_{n}\left(v_{i}\right)$ and find that the set $B$ of elements $x^{v^{*}-v_{i}} e_{i}$ form a basis for $F_{v^{*}}$. Moreover, this basis is adapted to the subspaces $G_{v}$ in the sense that the set $B_{v}=\left\{e_{i} \mid v_{i} \leq v\right\}$ is a basis for $G_{v}$. Under this identification, elements in $\operatorname{ARR}_{\xi, \delta}(F)$ are identified with families of subspaces $L_{v}^{*} \subseteq F_{v^{*}}$ so that the following conditions hold:

1. $L_{v}^{*} \subseteq G_{v}$ for all $v$.

2. If $v \leq v^{\prime}$, then $L_{v}^{*} \subseteq L_{v^{\prime}}^{*}$.

3. $\operatorname{dim}_{k}\left(L_{v}^{*}\right)=\delta(v)$.

4. $\operatorname{dim}_{k}\left(L_{v}^{*} / \sum_{v^{\prime}<v} L_{v^{\prime}}^{*}\right)=\alpha(v)$ for all $v \in V$.

We next partition the set $B$ by letting

$$
B(v)=B_{v}-\bigcup_{v^{\prime}<v} B_{v^{\prime}}
$$

and noting that

$$
B=\coprod_{v \in V} B(v) .
$$

Impose a total order on $V$, and then for each $v \in V$, select a total ordering on $B(v)$ to obtain a total ordering on the entire set $B$. Using the same total ordering on $V$, obtain an ordered basis $\mathcal{B}$ for the vector space $\bigoplus_{v \in V} W_{v}$ by choosing the standard ordered basis for each copy of $k^{\delta(v)}$ and using the given isomorphisms $W_{v} \cong k^{\delta(v)}$ to obtain a basis for $W_{v}$. This basis decomposes as

$$
\mathcal{B}=\coprod_{v \in V} \mathcal{B}(v)
$$

as well, with $\mathcal{B}(v)$ consisting of the basis elements for the copy of $W_{v}$ corresponding to $v$. For each $v \in V$, we define the $N \times \delta(v)$ matrix $M_{v}$ to be the matrix of the embedding $j_{v}$ relative to the bases $\mathcal{B}(v)$ for $W_{v}$ and $B$. We note that this matrix has a decomposition into blocks corresponding to the summands $\operatorname{span}(B(v))$, and the block corresponding to $B(v)$ and $\mathcal{B}\left(v^{\prime}\right)$ is identically zero if $v \not \leq v^{\prime}$. We next assemble the matrices $M_{v}$ into a total matrix $M$ by setting

$$
M=\left[M_{v_{1}}\left|M_{v_{2}}\right| \ldots \mid M_{v_{s}}\right] \text {, }
$$


where $\left\{v_{1}, v_{2}, \ldots, v_{s}\right\}$ is the total ordering on the set $V$. We obtain an $N \times D$ matrix of elements of $k$, where $D=\sum_{v} \delta(v)$. The group action described above of the group $\prod_{v \in V} \mathrm{GL}\left(W_{v}\right)$ on the set of frames can be interpreted as multiplication on the right by $\prod_{v \in V} \mathrm{GL}_{\delta(v)}(k)$ on the corresponding matrix, and we have therefore interpreted the set of all families of subspaces $\left\{L_{v}\right\}_{v \in V}$ with $L_{v} \subseteq F_{v}$ and $\operatorname{dim}_{k}\left(L_{v}\right)=\delta(v)$ for all $v$, as the orbit space of the algebraic group $\prod_{v \in V} \mathrm{GL}_{\delta(v)}(k)$ acting on the quasiprojective variety of all $N \times D$ matrices so that the rank of each submatrix $M_{v}$ is full, i.e., $=\delta(v)$, and so that the block of the matrix $M_{v}$ corresponding to the subset $B\left(v^{\prime}\right) \subseteq B$ is identically zero whenever $v^{\prime} \not \leq v$. It is a consequence of geometric invariant theory, as expounded in [16], that since this action has closed orbits and satisfies the stability hypothesis in [16], that the action admits a geometric quotient, and that therefore the set of orbits is naturally a quasiprojective variety.

Of course, $\mathbf{A R R}_{\xi, \delta}(F)$ is in general a proper subset of this variety, and we wish to verify that those conditions defining it are algebraic in nature. The strategy will be to construct the corresponding algebraic conditions on the variety $\mathcal{F}(F)$, observe that the conditions are invariant under the group action, and conclude that the orbit of the subspace is itself a quasiprojective variety. We enumerate the conditions one by one and describe the corresponding conditions on the space of frames:

1. $L_{v}^{*} \subseteq G_{v}$ for all $v$ : This condition is already accounted for with the requirement that the blocks of $M_{v}$ corresponding to the set $B\left(v^{\prime}\right)$ is zero if $\mathrm{V}$.

2. If $v \leq v^{\prime}$, then $L_{v}^{*} \subseteq L_{v^{\prime}}^{*}$ : This condition can be reinterpreted as the requirement that the $N \times\left(\delta(v)+\delta\left(v^{\prime}\right)\right)$ matrix

$$
\mu\left(v, v^{\prime}\right)=\left[M_{v} \mid M_{v^{\prime}}\right]
$$

has rank $\delta\left(v^{\prime}\right)$, or equivalently that all its $\left(\delta\left(v^{\prime}\right)+1\right) \times\left(\delta\left(v^{\prime}\right)+1\right)$ minors vanish. This is clearly an algebraic condition, invariant under the group action.

3. $\operatorname{dim}_{k}\left(L_{v}^{*}\right)=\delta(v)$ : This condition is already accounted for in the injectivity condition defining the variety of frames.

4. $\operatorname{dim}_{k}\left(L_{v}^{*} / \sum_{v^{\prime}<v} L_{v^{\prime}}^{*}\right)=\alpha(v)$ for all $v \in V$ : This condition can be reinterpreted as the requirement that the $N \times\left(\sum_{v^{\prime}<v} \delta\left(v^{\prime}\right)\right)$ matrix

$$
\lambda(v)=\left[M_{v_{1}^{\prime}}\left|M_{v_{2}^{\prime}}\right| \ldots \mid M_{v_{j}^{\prime}}\right]
$$

where $\left\{v_{1}^{\prime}, \ldots, v_{j}^{\prime}\right\}$ is an enumeration of all $v_{i}$ 's for which $v_{i}<v$, has rank exactly $\delta(v)-\alpha(v)$. This means that this set can be obtained as the action invariant Zariski closed set for which all the $(\delta(v)-\alpha(v)+1) \times(\delta(v)-\alpha(v)+1)$ minors of $\lambda(v)$ vanish, and removing from it the invariant closed Zariski closed set for which all the $(\delta(v)-\alpha(v)) \times(\delta(v)-\alpha(v))$ minors vanish.

We now let $\mathcal{F}_{0}(F) \subseteq \mathcal{F}(F)$ denote the quasiprojective variety of all matrices satisfying the above conditions. The set of orbits of $\mathcal{F}_{0}(F)$ is clearly in bijective correspondence with the subset $\operatorname{ARR}_{\xi, \delta}(F) \subseteq \mathcal{E}$. $\mathcal{E}$ is well known to be a projective variety. If we let $\mathcal{E}^{\prime} \subseteq \mathcal{E}$ denote the set of points in the image of frames satisfying condition (2) and the closed condition in (4) above, it is clear that $\mathcal{E}^{\prime}$ is a projective variety. Once we remove the set of points corresponding to frames in the second part of condition (4), we therefore obtain a quasiprojective variety, which is the desired result. 
Fig. 4 Visualization of $\xi_{0}$ and $\xi_{1}$ on $\mathbb{N}^{2}$ for our example, with the elements of the latter circled

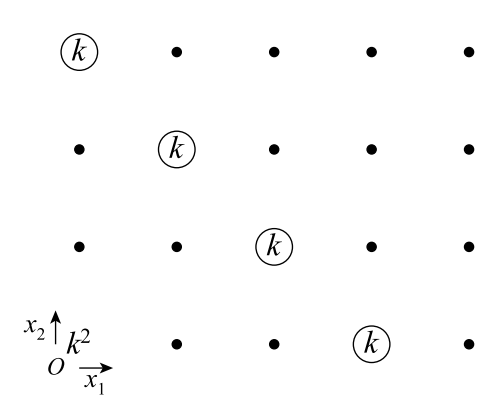

\subsection{The Continuous Invariant}

To further appreciate the complexity of the continuous invariant, we show its structure for a simple two-dimensional example. Suppose that we have a set of modules for which

$$
\begin{aligned}
& \xi_{0}=\{((0,0), 2)\}, \\
& \xi_{1}=\{((3,0), 1),((2,1), 1),((1,2), 1),((0,3), 1)\},
\end{aligned}
$$

as visualized on $\mathbb{N}^{2}$ in Fig. 4. It is easy to build a bifiltered simplicial complex whose first homology groups correspond to this picture. At $(0,0)$, we have a complex composed of two loops, giving us $k^{2}$. In each of the circled coordinates, we choose a sew a surface between the two loops such that no two complexes are sewn the same. For example, we could sew a cylinder at $(3,0)$, a punctured crosscap at $(2,1)$, and so on. Observe that the discrete invariants $\xi_{0}, \xi_{1}$ cannot discern the difference between the resulting complexes.

To obtain the classification, we apply Theorem 9. The generators of $F\left(\xi_{0}\right)$ are co-located, so we have the full group of automorphisms

$$
\mathrm{GL}\left(F\left(\xi_{0}\right)\right)=\mathrm{GL}\left(k^{2}\right)=\mathrm{GL}_{2}(k),
$$

where $\mathrm{GL}_{2}(k)$ is the group of invertible $2 \times 2$ matrices with elements from $k$. We use (3) to endow $\mathcal{R F}\left(\xi_{0}, \xi_{1}\right)$ with a variety structure. For each $(v, i) \in \xi_{1}, F\left(\xi_{0}\right)_{v}$ is isomorphic to $k^{2}$, and $\operatorname{dim} F\left(\xi_{1}\right)_{v}=1$, so $\operatorname{Gr}_{\operatorname{dim} F\left(\xi_{1}\right)_{v}}\left(F\left(\xi_{0}\right)_{v}\right)=\operatorname{Gr}_{1}\left(k^{2}\right)=\mathbb{P}^{1}(k)$, where $\mathbb{P}^{1}(k)$ denotes projective line, the set of lines in $k^{2}$ going through the origin. Then, the variety is simply $\mathbb{P}^{1}(k)^{4}$, as there are no containment conditions. The classification is given by the orbit space

$$
\mathrm{GL}_{2}(k) \backslash \mathbb{P}^{1}(k)^{4},
$$

where elements $g \in \mathrm{GL}_{2}(k)$ act in the evident way on the four lines, transforming each line to another.

We claim that no discrete invariant is possible for this bifiltration. Consider the subspace $\Omega$ of the orbit space containing pairwise-distinct lines. That is, we have four tuple of lines $\left(l_{1}, l_{2}, l_{3}, l_{4}\right)$, where $l_{i} \neq l_{j}$ for $i \neq j$. The subspace $\Omega$ is clearly invariant under the $\mathrm{GL}_{2}(k)$ action, and hence the orbit space $\mathrm{GL}_{2}(k) \backslash \Omega$ is a subspace of our orbit space in (4). Using matrices from $\mathrm{GL}_{2}(k)$, we transform the lines so that: 
1. $l_{1}$ becomes the $x$-axis;

2. $l_{2}$ becomes the $y$-axis, and

3. $l_{3}$ becomes the diagonal line spanned by $(1,1)$.

These transformations exist as $l_{1}, l_{2}$ span $k^{2}$, being nonzero and distinct, and $l_{3}$ cannot be zero or either axis after the first two transformations. We now have a tuple ( $x$-axis, $y$-axis, diagonal, $\lambda_{4}$ ), where $\lambda_{4}$ is $l_{4}$ after the transformations. While there are different matrices in $\mathrm{GL}_{2}(k)$ that can transform the original tuple to this tuple, the matrices differ by multiplication by a diagonal matrix, since the only matrices that preserve the axes and the diagonal line are diagonal matrices. Consequently, $\lambda_{4}$ is determined uniquely, and we may identify the orbits $\mathrm{GL}_{2}(k) \backslash \Omega$ with the lines in $\mathbb{P}^{1}(k)$ with the axes and the diagonal removed. Each such line is determined by its slope which cannot be $0, \infty$, or 1 , according to the discussion. Therefore, $\mathrm{GL}_{2}(k) \backslash \Omega$ can be identified with $\mathbb{P}^{1}(k)-\{0,1, \infty\}=k-\{0,1\}$.

Now, note that this classification is dependent on the field of coefficients $k$. If $k$ is uncountable, so is the subspace, and in turn, the full orbit space. If $k$ is a finite field, such as $\mathbb{F}_{p}$ for $p$ a prime, we get a finite solution for the subspace $\Omega$ we have chosen, but we still have not detailed the full picture for the orbit space. However, we already see the field-dependence problem: Changing the field not only changes the classification but also the target of the classification: We not only get different values, we get values from different sets altogether. This is analogous to getting Betti numbers in $\mathbb{Z}_{2}$ when computing over $\mathbb{Z}_{2}$, Betti numbers in $\mathbb{Z}_{3}$ when computing over $\mathbb{Z}_{3}$, and so on. Therefore, we cannot get a discrete invariant for our example.

\subsection{Refinement}

We have illustrated that our goal—obtaining a complete discrete invariant-is not attainable for multigraded objects. Intuitively, the continuous invariant captures subtle second-order information about the complicated transitions in a multigraded module. This information may be worthy of study, and we end this section by suggesting possible avenues of attack.

Our two discrete invariants may be viewed as the first two in a family of discrete invariants. We may develop standard homological algebra in the category of graded modules over an $n$-graded $k$-algebra $A_{n}$, with the resulting derived functors $\otimes_{A_{n}}$ and $\operatorname{Hom}_{A_{n}}$ now being equipped with the structure of an $n$-graded $A_{n}$-module [19]. In particular, the functor $\operatorname{Tor}_{i}^{A_{n}}(M, k)$ makes sense, and we now define a family of $n$ discrete invariants by

$$
\xi_{i}=\xi\left(\operatorname{Tor}_{i}^{A_{n}}(M, k)\right) \text {. }
$$

The first two invariants in the family match our two discrete invariants in the previous section. It may be interesting to study the rest of this family as each invariant will make the classification finer, as done recently by Knudson [14]. However, the existence of the continuous invariant indicates that no matter how many of these invariants we include, there will still be a residual continuous component in the classification.

While the set of orbits is not a variety, we conjecture that additional structure exists in the following form. Let $G=\operatorname{GL}\left(F\left(\xi_{0}\right)\right)$ and suppose that there is a family of closed subvarieties $\mathcal{R} \mathcal{F}_{n} \subseteq \mathcal{R} \mathcal{F}\left(\xi_{0}, \xi_{1}\right)$ such that: 
1. $\mathcal{R F}_{n} \subseteq \mathcal{R} \mathcal{F}_{n+1}$ for all $n$.

2. $\mathcal{R} \mathcal{F}_{n}$ is closed under the action of $G$.

3. $\mathcal{R} \mathcal{F}_{n}$ eventually becomes equal to $\mathcal{R} \mathcal{F}\left(\xi_{0}, \xi_{1}\right)$.

4. The set of orbits of the $G$-action on $\mathcal{R} \mathcal{F}_{n}-\mathcal{R} \mathcal{F}_{n-1}$ is an algebraic variety in a natural way.

This kind of structure is called an equivariant stratification of the variety in question, with the difference $\mathcal{R} \mathcal{F}_{n}-\mathcal{R F}_{n-1}$ being a stratum. The orbit varieties are called moduli spaces in classification problems for which the invariant lies in a given stratum. The result is known to hold in some special cases by the work of Cohen and Orlik [5] and Terao [18].

\section{The Rank Invariant}

Our study of multigraded objects shows that no complete discrete invariant exists for multidimensional persistence. We still desire a discriminating invariant that captures persistent information, that is, homology classes with large persistence. This information is not contained in our two discrete invariants, $\xi_{0}$ and $\xi_{1}$, as they capture birth and death coordinates of the generators in the complexes. What we need lies within the relationship between the two invariants or in the maps between the complexes. In this section, we propose and advocate a small and computable invariant that identifies persistent features in a multifiltration. Our invariant is equivalent to persistence barcodes, and therefore complete, for one-dimensional filtrations.

The persistent information is contained in the relating homomorphisms $\varphi_{u, v}$ in Definition 1. Recall that we incorporated these maps into a multigraded module through the action of the variables, requiring that $x^{v-u}: M_{u} \rightarrow M_{v}$ to be $\varphi_{u, v}$ in Definition 2. To analyze this family of maps, we begin by defining their domains.

Definition $5\left(\mathbb{D}^{n}\right)$ Let $\dot{\mathbb{N}}=\mathbb{N} \cup\{\infty\}$ with $u \leq \infty$ for all $u \in \dot{\mathbb{N}}$. Let $\mathbb{D}^{n} \subset \mathbb{N}^{n} \times \dot{\mathbb{N}}^{n}$ be the subset above the diagonal, $\mathbb{D}^{n}=\left\{(u, v) \mid u \in \mathbb{N}^{n}, v \in \dot{\mathbb{N}}^{n}, u \lesssim v\right\}$. For $(u, v),\left(u^{\prime}, v^{\prime}\right) \in \mathbb{D}^{n}$, we define $(u, v) \preceq\left(u^{\prime}, v^{\prime}\right)$ if $u \lesssim u^{\prime}$ and $v^{\prime} \lesssim v$.

It is easy to check that $\preceq$ is a quasi-partial order on $\mathbb{D}^{n}$. With this notation, our parameterization of singly-graded modules in Sect. 1.3 is a multiset from $\mathbb{D}^{1}$, and $\preceq$ indicates that the first pair contains the second when the pairs are viewed as intervals.

Definition 6 (Rank invariant $\rho_{M}$ ) Let $M$ be a finitely generated $n$-graded $A_{n}$-module. We define $\rho_{M}: \mathbb{D}^{n} \rightarrow \mathbb{N}$ to be $\rho_{M}(u, v)=\operatorname{rank}\left(x^{v-u}: M_{u} \rightarrow M_{v}\right)$.

The function $\rho_{M}$ is clearly a discrete invariant for $M$.

Lemma 1 (Order-preserving) If $(u, v) \preceq\left(u^{\prime}, v^{\prime}\right)$, then $\rho_{M}(u, v) \leq \rho_{M}\left(u^{\prime}, v^{\prime}\right)$, that is, $\rho_{M}$ is an order-preserving function from $\left(\mathbb{D}^{n}, \preceq\right)$ to $(\mathbb{N}, \leq)$.

Proof Immediate using the fact that given any composite $f \circ g$ of linear transformations, we have

$$
\operatorname{rank}(f \circ g) \leq \operatorname{rank} f \text {, rank } g \text {. }
$$


Fig. 5 The intervals of a barcode $\xi$ are drawn below the $t$-axis. Each interval $\left(t_{0}, t_{1}\right)$ defines a triangle as shown. The rank function $\vartheta(\xi)(t, s)$ is the number of triangles that contain $(t, s)$

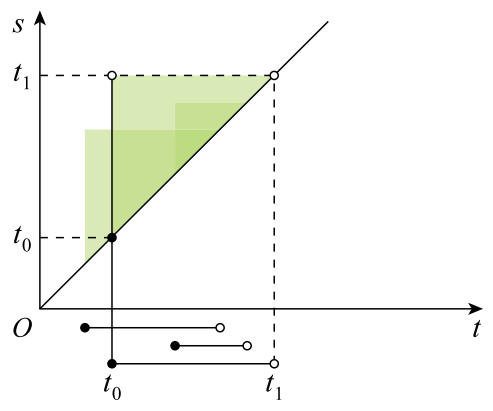

We now state the rank invariant's completeness in one dimension through its equivalence to barcodes. We note that the following theorem is the converse of the $k$-triangle lemma $[9,21]$.

Theorem 12 (Completeness) The rank invariant $\rho_{M}$ is complete for singly-graded modules $M$.

Proof To prove completeness, we show equivalence via a bijection $\vartheta$ between the set of barcodes and the set of rank invariants. According to the classification theorem for a graded module $M$ recalled in Sect. 1.3, the intervals in its barcode $\xi$ capture the lifetimes of the generators of $M$. Therefore, the corresponding rank function is

$$
\vartheta(\xi)(t, s)=\operatorname{card}\left\{\left(\left(t^{\prime}, s^{\prime}\right), i\right) \in \xi \mid(t, s) \subseteq\left(t^{\prime}, s^{\prime}\right)\right\} .
$$

Figure 5 illustrates this correspondence. The barcode intervals are drawn below the $t$ axis and the rank function's domain, $\mathbb{D}^{1}$, exists above the diagonal in the $(t, s)$-plane. Each interval $\left[t_{0}, t_{1}\right)$ has a triangular region defined by inequalities $t \geq t_{0}, s<t_{1}$, and $s \geq t$, with corner vertex $\left(t_{0}, t_{1}\right)$ and vertices $\left(t_{0}, t_{0}\right)$ and $\left(t_{1}, t_{1}\right)$ on the diagonal. Half-infinite intervals correspond to degenerate triangles, but they are handled easily, so we do not discuss them here. The rank function $\vartheta(\xi)(t, s)$ is simply the number of triangles that contain $(t, s)$. As an aside, we note that the map $(t, s) \mapsto(t, s-t)$ gives the index-persistence figures in the previous papers $[9,21]$.

Clearly, we can construct each triangle from its corner by projecting the corner vertically and horizontally onto the diagonal. Moreover, there is a trivial bijection between the corner $\left(t_{0}, t_{1}\right)$ and the interval $\left[t_{0}, t_{1}\right)$. Given a barcode $\xi$, we know how to build the rank function $\vartheta(\xi)$ by the equation above. Given a rank function $\rho$, we need to identify the corner points to build the corresponding barcode. We begin by first walking along the diagonal until the rank function is nonzero at $t_{0}=\arg \min _{t} \rho(t, t) \neq 0$. By Lemma 1 , the function $s \mapsto \rho\left(t_{0}, s\right)$ is a nonincreasing function, so we walk vertically up until $t_{1}$ where $\rho\left(t_{0}, t_{1}\right)<\rho\left(t_{0}, t_{0}\right)$. The point $\left(t_{0}, t_{1}\right)$ is a corner, so we subtract its triangle from $\rho$. The proof follows by induction.

When the module is the persistence module associated to the $i$ th homology of a multifiltration, we can define the rank invariant directly in terms of the input. 
Definition $7\left(\rho_{X, i}\right)$ Let $X=\left\{X_{v}\right\}_{v \in \mathbb{N}^{n}}$ be a multifiltration. We define $\rho_{X, i}: \mathbb{D}^{n} \rightarrow \mathbb{N}$ over field $k$ to

$$
\rho_{X, i}(u, v)=\operatorname{rank}\left(H_{i}\left(X_{u}, k\right) \rightarrow H_{i}\left(X_{v}, k\right)\right) .
$$

The function $\rho_{X, i}$ is a homeomorphism invariant of the multifiltered space, deriving its invariance from the invariance of $\rho_{M}$. Intuitively, Theorem 12 means that the rank invariant for one-dimensional filtrations may be separated into a set of overlapping triangles whose thickness at any point is the rank. These triangles, in turn, carry the same information as a set of intervals or the barcode. Our classification theorem, on the other hand, implies that a similar result is not possible for higher dimensions. As our example in Sect. 5.2 illustrates, the picture is much more complicated: It is not possible to separate the rank invariant into overlapping regions to extend the barcode.

The rank invariant does extend, however, as an incomplete invariant. We may utilize it to identify persistent features by the following procedure. Given a rank invariant, we look for points $(u, v) \in \mathbb{D}^{n}$ that are far from the diagonal and have a neighborhood of constant value. The first condition corresponds to the persistence of the features. The second condition indicates the stability of our choice $(u, v)$. With this procedure, the rank invariant emerges as a practical tool for reliable estimation of the Betti numbers of multifiltered spaces.

\section{Conclusion}

We believe that the primary contribution of this paper is the theoretical description of the structure of algebraic multidimensional persistence: We identify the corresponding algebraic structure, classify it, and undertake its parameterization. Our theory reveals that a complete discrete invariant does not exist for multidimensional persistence, unlike its one-dimensional counterpart. A second practical contribution of our paper is the rank invariant, a tool for robust estimation of the Betti numbers. We prove that the rank invariant is equivalent to the persistent barcode in one dimension, so it is complete when it can be. Unlike the barcode, the rank invariant extends to higher dimensions as an incomplete but useful invariant.

We have developed an algorithm for computing the rank invariant. For bifiltrations, the rank invariant is already four-dimensional, so we are examining possible interfaces for visualizing and exploring the rank invariant. We plan to apply our work toward automatic identification of features in multifiltrations, such as the filtered tangent complex [6].

\section{References}

1. Atiyah, M.F., Macdonald, I.G.: Introduction to Commutative Algebra. Addison-Wesley, Reading (1969)

2. Carlsson, G., Zomorodian, A., Collins, A., Guibas, L.J.: Persistence barcodes for shapes. Int. J. Shape Model. 11(2), 149-187 (2005)

3. Carlsson, G., Ishkhanov, T., de Silva, V., Zomorodian, A.: On the local behavior of spaces of natural images. Int. J. Comput. Vis. 76(1), 1-12 (2008) 
4. Chazal, F., Lieutier, A.: Weak feature size and persistent homology: computing homology of solids in $\mathbb{R}^{n}$ from noisy data samples. In: Proceedings of ACM Symposium on Computational Geometry, pp. 255-262 (2005)

5. Cohen, D.C., Orlik, P.: Gauss-Manin connections for arrangements I. Eigenvalues. Compos. Math. 136(3), 299-316 (2003)

6. Collins, A., Zomorodian, A., Carlsson, G., Guibas, L.: A barcode shape descriptor for curve point cloud data. Comput. Graph. 28, 881-894 (2004)

7. de Silva, V., Carlsson, G.: Topological estimation using witness complexes. In: Proceedings of IEEE/Eurographics Symposium on Point-Based Graphics, pp. 157-166 (2004)

8. de Silva, V., Ghrist, R., Muhammad, A.: Blind swarms for coverage in 2-D. In: Proceedings of Robotics: Science and Systems. http://www.roboticsproceedings.org/rss01/ (2005)

9. Edelsbrunner, H., Letscher, D., Zomorodian, A.: Topological persistence and simplification. Discrete Comput. Geom. 28, 511-533 (2002)

10. Frosini, P., Mulazzani, M.: Size homotopy groups for computation of natural size distances. Bull. Belg. Math. Soc. Simon Stevin 6(3), 455-464 (1999)

11. Ghrist, R.: Barcodes: the persistent topology of data. Bull. Am. Math. Soc. New Ser. 45(1), 61-75 (2008)

12. Gromov, M.: Hyperbolic groups. In: Gersten, S. (ed.) Essays in Group Theory, pp. 75-263. Springer, New York (1987)

13. Gyulassy, A., Natarajan, V., Pascucci, V., Bremer, P.T., Hamann, B.: Topology-based simplification for feature extraction from 3D scalar fields. In: Proceedings of IEEE Visualization, pp. 275-280 (2005)

14. Knudson, K.P.: A refinement of multi-dimensional persistence. Homotopy Homol. Appl. 10, 259-281 (2008)

15. Lee, A., Mumford, D., Pedersen, K.: The nonlinear statistics of high-contrast patches in natural images. Int. J. Comput. Vis. 54(1-3), 83-103 (2003)

16. Mumford, D., Fogarty, J., Kirwan, F.: Geometric Invariant Theory, 3rd edn. Ergebnisse der Mathematik und ihrer Grenzgebiete (2), vol. 34. Springer, Berlin (1994)

17. Serre, J.-P.: Local Algebra. Springer, Berlin (2000)

18. Terao, H.: Moduli space of combinatorially equivalent arrangements of hyperplanes and logarithmic Gauss-Manin connections. Topol. Appl. 118(1-2), 255-274 (2002)

19. Weibel, C.A.: An Introduction to Homological Algebra. Cambridge Studies in Advanced Mathematics, vol. 38. Cambridge University Press, Cambridge (1994)

20. Zomorodian, A.: Computational topology. In: Atallah, M., Blanton, M. (eds.) Algorithms and Theory of Computation Handbook, 2nd edn. CRC, Boca Raton (2009) (in press)

21. Zomorodian, A., Carlsson, G.: Computing persistent homology. Discrete Comput. Geom. 33(2), 249274 (2005) 